\title{
G Research Square $=15$ \\ Enhanced the light absorptance of stain-etched black Silicon decorated by TiN nanoparticles
}

\section{Kaiqiang Wang}

University of Electronic Science and Technology of China

Shuang Liu ( $\nabla$ shuangliu@uestc.edu.cn )

University of electronic and Science Technology of China https://orcid.org/0000-0002-9824-3734

Jiacheng Li

University of Electronic Science and Technology of China

Shenglan Wu

University of Electronic Science and Technology of China

Jiankai Xia

University of Electronic Science and Technology of China

Jinping Chen

University of Electronic Science and Technology of China

Chunhui Tian

University of Electronic Science and Technology of China

Yong Liu

University of Electronic Science and Technology of China

Zhiyong Zhong

University of Electronic Science and Technology of China

\section{Research Article}

Keywords: Titanium nitride nanoparticles, Black Silicon, Stain etching, PIN photoelectronic detector

Posted Date: February 12th, 2021

DOI: https://doi.org/10.21203/rs.3.rs-219778/v1

License: (c) (i) This work is licensed under a Creative Commons Attribution 4.0 International License.

Read Full License

Version of Record: A version of this preprint was published at Journal of Materials Science: Materials in Electronics on April 22nd, 2021. See the published version at https://doi.org/10.1007/s10854-021-05724W. 


\section{Abstract}

Due to the high energy, narrow distribution and breaking through the absorption limitation, plasmon induced hot electrons has been widely applied to extend the photoresponse spectra of the semiconductor. In order to further enhance the resonance effect of local plasmon based on metallic nanostructures, we used hydrofluoric stain etching method to fabricate nanostructured black silicon (BSi) and deposited titanium nitride ( $\mathrm{TiN}$ ) nanoparticles on its surface by reactive magnetron-sputtering. The results show that the BSi modified by plasmonic TiN nanoparticles has higher absorption in wavelength range from 1100 to $2500 \mathrm{~nm}$ compared to that of conventional acid etching of BSi. A PIN photoelectronic detector fabricated by the proposed BSi shows excellent device performance with responsivity of $0.45 \mathrm{~A} / \mathrm{W}$ at $1060 \mathrm{~nm}$ in near infrared band.

\section{Introduction}

As well-known as, Silicon is widely used in various optoelectronic devices such as photoelectronic detectors, solar cells, biochemical sensors. But the inherent band gap width of silicon material $(\mathrm{Eg}=$ $1.12 \mathrm{eV}$ ) limits its application in near-infrared band. Black silicon refers to a surface modification of silicon where a nanoscale surface structure is formed through etching and can significantly reduce the reflectance of incident photos due to light trapping effect [1, 2]. BSi prepared by femtosecond laser pulses in $\mathrm{SF}_{6}$ environment has great light absorption in the wavelength range from 250 to $2500 \mathrm{~nm}$ due to the impurity band gap levels induced by the doped chalcogen [3-5]. Texturing silicon surface by laser ablation requires a lot of time and economic cost. Thus, more attention has been paid on other technical means for the fabrication of BSi, such as electrochemical etching [6], reactive ion etching (RIE) [7], stain etching [8,9], metal-assisted etching [10], etc. . However, BSi materials generated in these approaches wouldn't break the limitation of the large energy bandgap of Si without heavily doped impurity elements, which means the electromagnetic wave with a wavelength over $1100 \mathrm{~nm}$ won't generate electro-holes pairs inside semiconductor [11].

Recently, constructing metallic nanostructures onto semiconductor materials to generate surface plasmon (SP) provides an unprecedented technical route for manipulating of light at the nanoscale [12, 15]. SPs are coherent oscillations of free electrons in metal coupled to incident electromagnetic fields which are categorized into propagating surface plasmons (PSPS) and localized surface plasmons (LSPs) $[16,20]$. Following optical absorption and excitation, SPs can transport energy to hot electrons through nonradiative decay process. In this case, these hot electrons can be injected into the conduction band of semiconductor before thermalization [14-19]. And the energy of Schottky barrier formed by metalsemiconductor contact gets much lower, which means low-energy electrons can jump to the conduction band level and result in photocurrent more easily without crossing bandgap of semiconductor [17, 20]. Moreover, the spectral window of photodetectors can be tuned by regulating the shape and physical dimension of the nanostructures. Variety of structures have been studied including nano wires [21], nano cube [22], and nano hole [23] in previous researches. However, these achievements usually focus on a narrowed absorption peak and the fabrication of these subwavelength nanostructures usually involves in 
costly lithography $[18,20,22]$. It's still a challenge to realize a Si-based device with efficient absorption in a wide range including near-infrared region by the cheap and simple configuration.

In this article, we are motivated to proposed a plasmon-enhanced absorber characterized by high absorption in a broadband from visible to near-infrared range and its application in silicon based photoelectronic detector. We use stain-etched method to fabricated BSi with hydrofluoric acid (HF) and sputter Titanium target on the microstructural surfaces in nitrogen where the high-resolution lithography is not necessary. The influence of different geometry of $\mathrm{TiN}$ nanoparticles on light absorbance was studied in the wavelength range of 350-2500 nm, and the PIN photoelectronic detector based on this LSPs-BSi composite absorber has been extensively investigated with an emphasis on device responsivity at $1060 \mathrm{~nm}$.

\section{Methods}

Double polished n-type (100) silicon wafers with a thickness of $675 \mu \mathrm{m}$ and a resistivity of $2500-3000 \Omega$ $\mathrm{cm}$ were utilized as substrates. At first, the Si wafers were ultrasonically cleaned by acetone, ethanol, distill water in turns, and then immersed into diluted HF acid solution(5 wt\%) for 30 minutes to remove the primary oxide layer. Before acid corrosion, the previously silicon tablets were roughed and thinned to about $300 \mu \mathrm{m}$ by physical grinding with $\mathrm{SiO}_{2}$ particles $20 \mathrm{~nm}$ to $100 \mathrm{~nm}$ in diameter. Stain etching method employs $40 \% \mathrm{HF}$ and $65 \% \mathrm{HNO}_{3}$ by a ratio of 5 to 1 to perform an anisotropic chemical wet etching at room temperature $\left(\sim 25^{\circ} \mathrm{C}\right)$. Moreover, to realize SPs, the TiN films were coated on the prepared BSi substrates at a rate of $2 \mathrm{~nm} / \mathrm{s}$ in a nitrogen condition by reactive magnetron sputtering at the working pressure of $10^{-3} \mathrm{~Pa}$. The reflectance $(R)$ and transmittance $(T)$ of prepared samples were determined by spectrophotometer. And the absorptance $(A)$ was calculated by formula $A=1-T-R$.

Considering the elements and technical flow process of Si-PIN photoelectronic detector, we finally introduced LSPs-BSi composite absorption layer by the way of back incidence. Figure 1(b) shows the structure of PIN photoelectronic device and the front side of detector was processed in accordance with the standard flow sheet processing technology. First, a p-typed layer was fabricated by thermal diffusion of boron on the front side of polished n-typed silicon. Second, depositing $\mathrm{Si}_{3} \mathrm{~N}_{4}$ on the photosensitive area as antireflection film for near-infrared bands and acting as a passivation layer in a meanwhile. Third, preparing BSi nanostructures on the back side of wafer as above steps and forming a P-doped $\mathrm{N}^{+}$layer through Phosphorus ion implantation. Finally, sputtering TiN film on BSi surface to perform the introduction of LSPs-BSi composite absorber. Figure 1(a) shows the array of detector unit on a 6 inches wafer after tape-out and the encapsulated device in the upper right corner. The morphologies of samples were observed by a field emission scanning electron microscope (SEM; FEI Quanta FEG 450). The light absorptance was obtained at room temperature using a spectrophotometer (PerkinElmer Lambda-750) equipped with a 150-mm integrating sphere detector. To test the performance of proposed detector, a platform consisting of a xenon lamp equipped with a grating monochromator $(200 \mathrm{~nm}-2500 \mathrm{~nm})$ and a Keithley 2400 apparatus was constructed. During testing, a reverse bias of about $180 \mathrm{~V}$ was applied to the 
detector by constant voltage source and the photocurrent $\mathrm{I}_{\mathrm{L}}$ was recorded. Each value of incident light power $P_{\text {in }}$ at different specific wavelength can be obtained worthwhile. The detector responsivity $R_{e}$ can

be calculated by formula $R_{e}=I_{L} / P_{i n}$. The dark current of the device was also observed in a black box made of Aluminum.

\section{Results And Discussion}

Stain etching method leads to formation of porous silicon and the main chemical reaction is results in a continus process of oxidation-followed-by-dissolution in which $\mathrm{HNO}_{3}$ is devoted to injecting holes and oxidizing silicon, and HF for the removal of the formed oxide [8, 9]. Figure 2 shows the typical SEM images of stain-etched black silicon in top view at $30^{\circ}$ angle for different etching time. It's clearly to see that etching appears first in defect and damage area caused by thinning step. As the reaction progress, physical scratches have been converted to dense bowl-shaped corrosion orifices in 5 minutes. And as the corrosion deepens, adjacent corrosion pits appear staggered overlap leading the formation of irregular bowl-shaped structures with a size of $0.8-2 \mu \mathrm{m}$ eventually.

The porosity of BSi rises with the increase of reaction time, but the effective numbers of reflections is greatly reduced due to the erosion in the sidewall of holes. As the results shows in Fig. 4, the average light absorptance of samples is promoted to $80 \%$ in visible range and in near-infrared band there is still an improvement of nearly $15 \%$ compared to untreated planar silicon. In the procedure of TiN nanoparticles growth, stain-etched BSi samples were deposited on ultrathin layer of titanium in nitrogen environment. The target material of experimental coating film is high purity titanium metal and deposition rate was controlled at $2 \mathrm{~nm}$ per second by adjusting the gas flow velocity and intensity of pressure. It can be known from the film kinetic growth process; the atoms of the incident material first form a stable nucleus on the substrate after absorbing or revaporization [24]. As the thickness of the surface layer increases, atoms coalesce at nucleation sites to form nanoislands and result in a continuous film finally. Since the uneven surface of stain-etched BSi, the original linear sedimentation path under the action of gravity in the vacuum chamber is disturbed and makes it more difficult to form a continuous membrane which called self-shadowing effects in obliquely deposited [25]. To ensure the formation of isolated TiN nanoparticles, deposition time was limited to 25 seconds and Fig. 3 shows the SEM images of TiN nanoparticles decorated BSi at different time points.

When depositing 5 seconds, the bottom and side walls of the corroded bowl hole are attached with TiN nanoparticles as shown in Fig. 3(a). And it is found that due to the shadowing effect of the black silicon microstructure during the deposition process, TiN after nucleation accumulation resulting in the formation of particles maximum size is not limited to $10 \mathrm{~nm}$, but distributed in the range of $10 \sim 25 \mathrm{~nm}$, and attachment location is relatively scattered. When the deposition time reaches 15 seconds, the metal particles become denser and appear partially continuous without significiantly change of particle size, nevertheless. In Fig. 3(c), granular deposits of TiN have been replaced by continuous TiN film with nonuniform projections. It also can be demonstrated by the absorption line in Fig. 4 that BSi/10 nm TiN basically retains the absorption characteristics of BSi in long wavelength ultraviolet band which means 
the coating materials does not form membrane structure. In contrast, BSi with 15 and $25 \mathrm{sec}$ deposition samples shows higher absorption due to low reflectivity of TiN materials to ultraviolet light. And the absorption rate decreased as the deposition thickness increases cause of the reflectance effect of TiN in 600-1100 nm. However, in the near infrared waveband(1100-2500 nm), the LSPs effects of TiN nanoparticles shows extensive enhancement of spectral absorption from $15 \%$ to nearly $65 \%$ compared to separate BSi materials. As for these samples shows lower performance as exhibited, this is because the continuous TiN film increases reflection of near-infrared light.

To further study the effect of the introduction of LSPs-BSi composite absorption layer on detector performance, we ended up dealing with it in the form of back incidence (structure of PIN detector as shown in Fig. 1(b)) and test the device in terms of spectral response range, responsivity and dark current. Figure 6 gives the results of PIN photodetector with BSi/10 nm TiN absorber, the responsivity of two different devices are re-plotted and exhibited for comparison. Device 1 is a commercial Si-PIN detector(S1336-44BK) and the parameter is taken from the public Website of Hamamatsu Photonics Company [26]. Device 2 is also a BSi-PIN detector forming light-trapping structures on back surface from reference [27]. It can be clearly seen that our newly fabricated detector has higher response after the wavelength exceeds $700 \mathrm{~nm}$ and shows satisfactory performance in near infrared band. The response rate was $0.46 \mathrm{~A} / \mathrm{W}$ at $1060 \mathrm{~nm}$ and $0.27 \mathrm{~A} / \mathrm{W}$ even at $1100 \mathrm{~nm}$. The LSPs-BSi composite detector peak wavelength of $980 \mathrm{~nm}$, responsivity of $0.64 \mathrm{~A} / \mathrm{W}$, even above the silicon band gap width of $1100 \sim$ $1170 \mathrm{~nm}$ peak also has more than $10 \%$ of the highest response, this part of the responsivity from the impurity level absorb photons, and plasmons materials hot electron collection detection results.

At less than $700 \mathrm{~nm}$ in visible spectrum, Device 3 shows a relatively low responsivity compared to commercial PIN detector. This decline is caused by two factors. Firstly, device 1 used an antireflection layer for infrared band on the front of the detector which lowers the incidence of visible light. The second, unlike the normal incidence of device 1, design of back-incidence structure (refer to Fig. 2) enables the photon in the visible band mainly absorbed and completing generation and recombination of carries in the $\mathrm{P}$ layer which hardly any photocurrent is produced. This interpretation also applies to the performance of device 2 which has analogous structure. Above $700 \mathrm{~nm}$ and near infrared band, the photon energy is able of penetrating $\mathrm{P}$ layer and absorbed by LSPs-BSi layer, and then lots of generated carriers can be collected under the action of reverse bias. In the meanwhile, due to the localized surface plasmons resonance effects of TiN nanoparticles and light trapping effects, the original indirect bandgap structure of silicon-based materials has been transformed to quasi-direct band gap which leads the peak response of the detector was redshifted. And the dark current of the detector under working condition is less than 10nA measured in the aluminum black box. From what has been discussed above, the deposition of ultrathin TiN film on rough black silicon surface can form nanoparticles with metal properties of random size and hybridizing LSP modes. The absorption layer is introduced into the PIN photodetector, which effectively improves the sensitivity of the near-infrared band.

\section{Conclusion}


In this paper, we utilized a TiN nanoparticle decorated BSi absorber with high property through a facile two-steps process, stain-etched and titanium deposition in nitrogen. It has strong light harvesting effects with an absorptivity of $80 \%$ in visible light and $70 \%$ in near infrared band. For further study in plasmonic enhanced optoelectronic device, a novel silicon-based PIN photoelectronic detector with composited absorption layer fabricated on the back surface has been prepared. Through the contrast of responsivity with commercial Si based PIN detector (S1336-44BK from website [27]) and similar structure of BSi detector [28], it is concluded that above absorbing layer has certain positive significance for the effective photoelectric conversion of near infrared light. The photoelectronic detector has a substantial increase in responsivity, particularly in the near infrared wavebands, rising to $0.46 \mathrm{~A} / \mathrm{W}$ at $1060 \mathrm{~nm}$ and $0.27 \mathrm{~A} / \mathrm{W}$ at $1100 \mathrm{~nm}$, respectively.

\section{Declarations}

\section{Acknowledgment}

This work was supported by the National Natural Science Foundation of China (Grant No.61734002, 61435010,61177035 and 61421002). The author would like to thank State Key Laboratory of Electronic Thin Films and Integrated Devices in China for the help and equipment support.

\section{References}

1. Liu X, Coxon P R, Peters M, Hoex B, Cole J M and Fray D J. Black silicon: fabrication methods, properties and solar energy applications. Energy Environ. Sci. 7, 3223-3263 (2014)

2. Burresi, F. Pratesi, F. Riboli, D.S. Wiersma, Complex photonic structures for light harvesting. Adv. Opt. Mater. 3, 722-743 (2015)

3. Wu C, Crouch $\mathrm{CH}$, Zhao L et al. Near-unity below-band-gap absorption by microstructured silicon. Appl Phys Lett 78(13):1850-1852(2001)

4. Xia Y, Liu B, Liu J, Shen Z and Li C, A novel method to produce black silicon for solar cells. Sol Energy. 85, 1574-1578 (2011)

5. Wen C, Yang HD, Li XH et al. Transmission electron microscopy investigation of crystalline silicon surface irradiated by femtosecond laser pulses in different background atmospheres. Applied Physics A 109(3):635-641(2012)

6. Kim, N. Cho, Morphological and nonstructural features of porous silicon prepared by electrochemical etching. Nanoscale Res. Lett. 7, 408 (2012)

7. Dussart, X. Mellhaoui, T. Tillocher, P. Lefaucheux, M. Volatier, C. Socquet-Clerc, P. Brault, P.Ranson. Silicon columnar microstructures induced by an SF6/02plasma. J. Phys. D. 38, 3395-3402 (2005)

8. Shih, K. Jung, T. Hsieh, J. Sarathy, J. Campbell and D. Kwong, Photoluminescence and Structure of Chemically Etched Si. Appl. Phys. Lett.15, 1863-1865 (1992) 
9. Su, S. Li, G. Zhao, Z. Wu, Y. Yang, W. Li, Y. Jiang, Optical properties of black silicon prepared by wet etching. J. Mater. Sci. Mater. Electron. 23, 1558-1561 (2012)

10. Venkatesan, M.K. Arivalagan, V. Venkatachalapathy, J.M. Pearce, J. Mayandi, Effects of silver catalyst concentration in metal assisted chemical etching of silicon. Mater. Lett. 221, 206- 210 (2018)

11. Casalino M, Coppola G, lodice M, Rendina I and Sirleto L. Near-infrared sub-bandgap all-silicon photodetectors: state of the art and perspectives Sensors .10, 10571-600 (2010)

12. Li W and Valentine J G, Harvesting the loss: surface plasmon-based hot electron photodetection Nanophotonics. 6, 177-191 (2017)

13. A. Atwater, A. Polman, Plasmonics for improved photovoltaic devices. Nat. Mater. 9, 205-213 (2010)

14. Brongersma $\mathrm{M} \mathrm{L}$, Halas $\mathrm{N} J$ and Nordlander P, Plasmon-induced hot carrier science and technology Nat. Nanotechnol. 10, 25-34 (2015)

15. L. Kelly, E. Coronado, L.L. Zhao, G.C. Schatz, The optical properties of metal nanoparticles: the influence of size, shape, and dielectric environment. J. Phys. Chem. B. 107, 668-677 (2003)

16. A. Willets, R. P. Van Duyne. Localized Surface Plasmon Resonance Spectroscopy and Sensing. Annual Review of Physical Chemistry, 2007, 58(1): 267-297.

17. Clavero C, Plasmon-induced hot-electron generation at nanoparticle/metal-oxide interfaces for photovoltaic and photocatalytic devices Nat. Photonics. 8, 95-103 (2014)

18. Knight M W, Sobhani H, Nordlander P and Halas N J, Photodetection with Active Optical Antennas.332, 702-4 (2011)

19. Knight M W, Wang $Y$, Urban A S, Sobhani A, Zheng B Y, Nordlander $P$ and Halas N, J Embedding, Plasmonic nanostructure diodes enhances hot electron emission Nano Lett. 13, 1687-92 (2013)

20. Sun, K. Ueno, H. Yu, et al. Direct imaging of the near field and dynamics of surface plasmon resonance on gold nanostructures using photoemission electron microscopy. Light: Science \& Applications: 13, 2: e118(2013)

21. Li, J. Valentine, Metamaterial Perfect Absorber Based Hot Electron Photodetection, Nano Lett. 14, 3510-3514. (2014)

22. Lin, K. T, Chen, H. L.; Lai, Y. S, Yu, C. C. Silicon-based broadband antenna for high responsivity and polarization-insensitive photodetection at telecommunication wavelengths. Nat. Commun. 5, 3288 (2014)

23. Wang, J. Gao, H. Yang, X. Wang, Near-infrared absorption enhancement in micro structured silicon by Ag film deposition. J. Mater. Sci. Mater. Electron. 27,9002-9007(2016)

24. Salomon, G. Bassou, H. Aourag, et al. Local excitation of surface plasmon polaritons at discontinuities of a metal film: Theoretical analysis and optical near-field measurements. Physical Review B, 65(125409) (2002)

25. Vick, L.J. Friedrich, S. K. Dew, et al. Self-shadowing and surface diffusion effects in obliquely deposited thin films[J]. Thin Solid Films. 339(1-2): 88-94.(1999) 
26. Hamamatsu Photonics Company. Light semiconductor detector. Silicon photoelectronic diode. http://www.hamamatsu.com.cn/product/category/ 10002/10003/10088/index.html 28.

27. Moloney AM, Wall L, Mathewson A et al. Novel black silicon PIN photodiodes//Integrated Optoelectronic Devices 2006. Int Soc Optics Photonics:61190B-61190B-8 (2006)

\section{Figures}
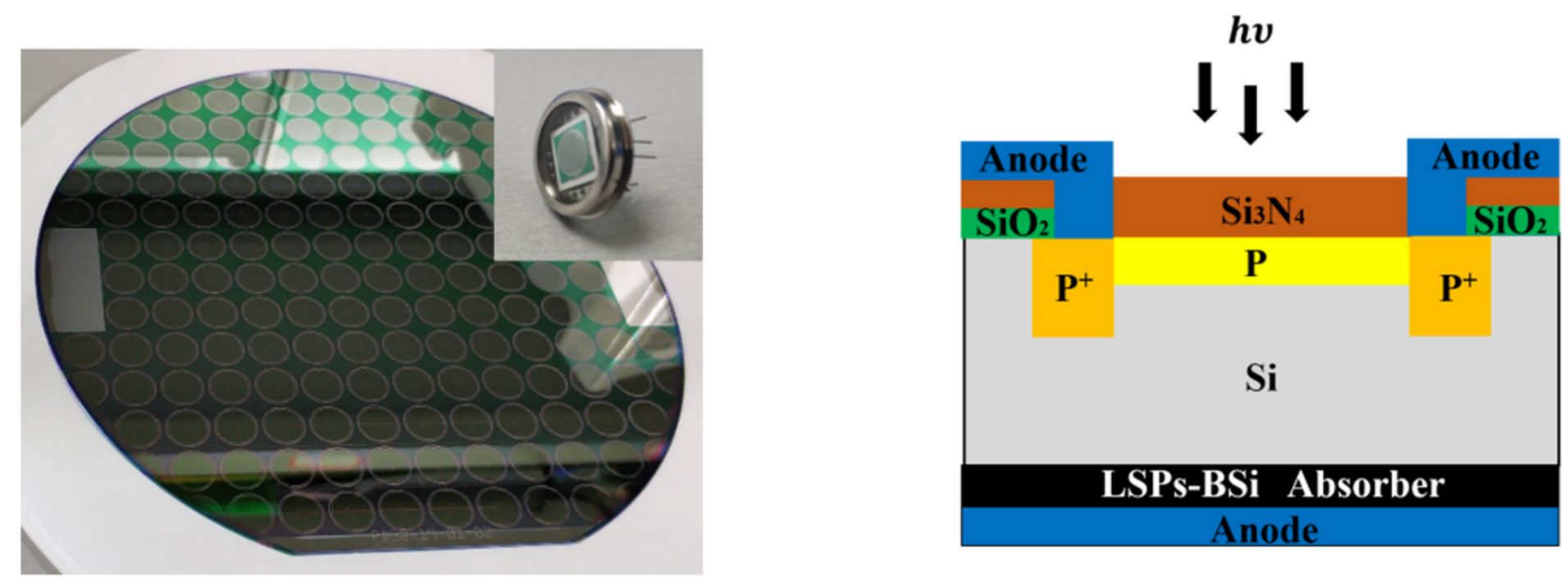

Figure 1

Real image of the detector (a) and structure of Si-PIN detector based on LSPs-BSi Absorber(b)

\section{(a) $1 \mathrm{~min}$}

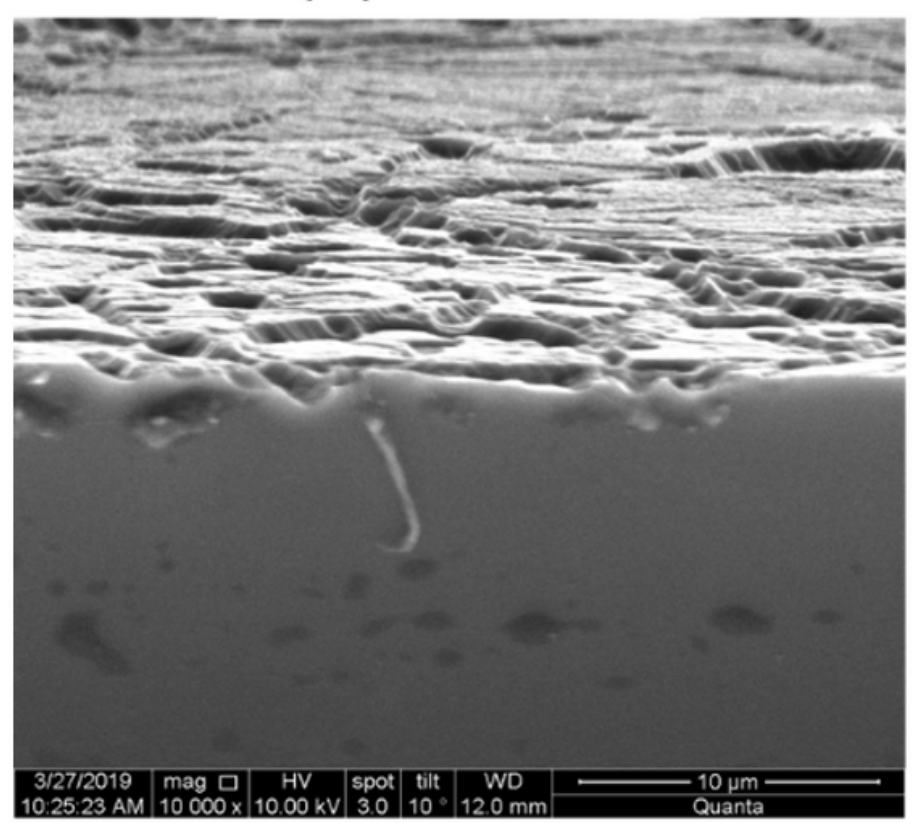

(b) 5 min

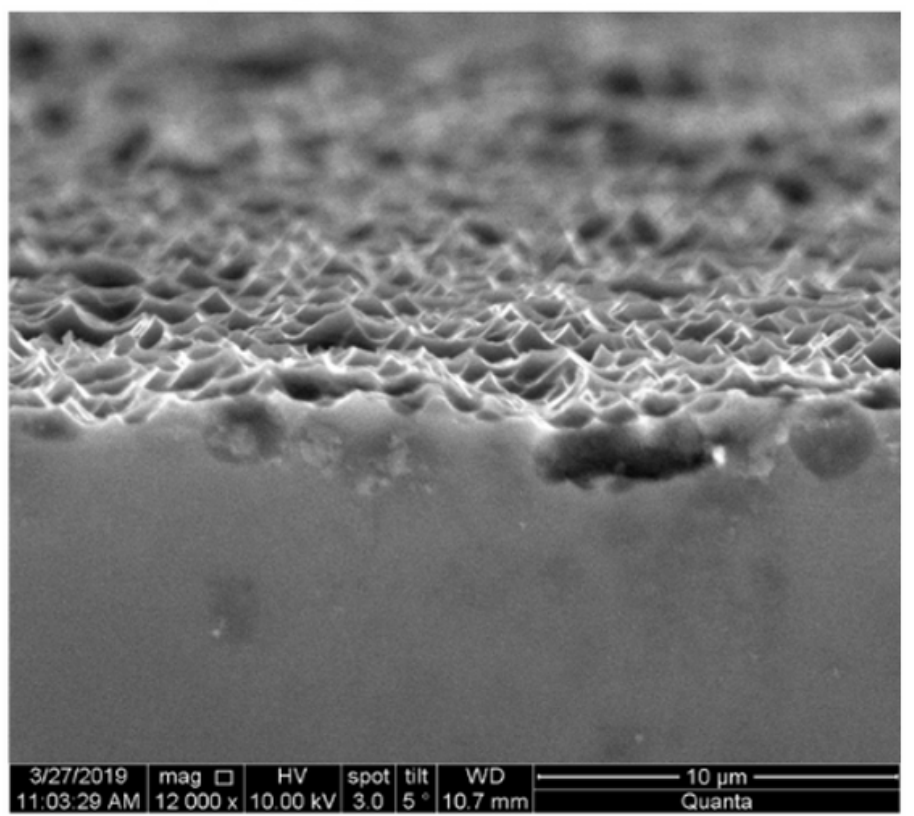

Figure 2 
SEM images of BSi microstructures manufactured by stain-etched method at various etching time, (a) $1 \mathrm{~min}$; (b) $5 \mathrm{~min}$.
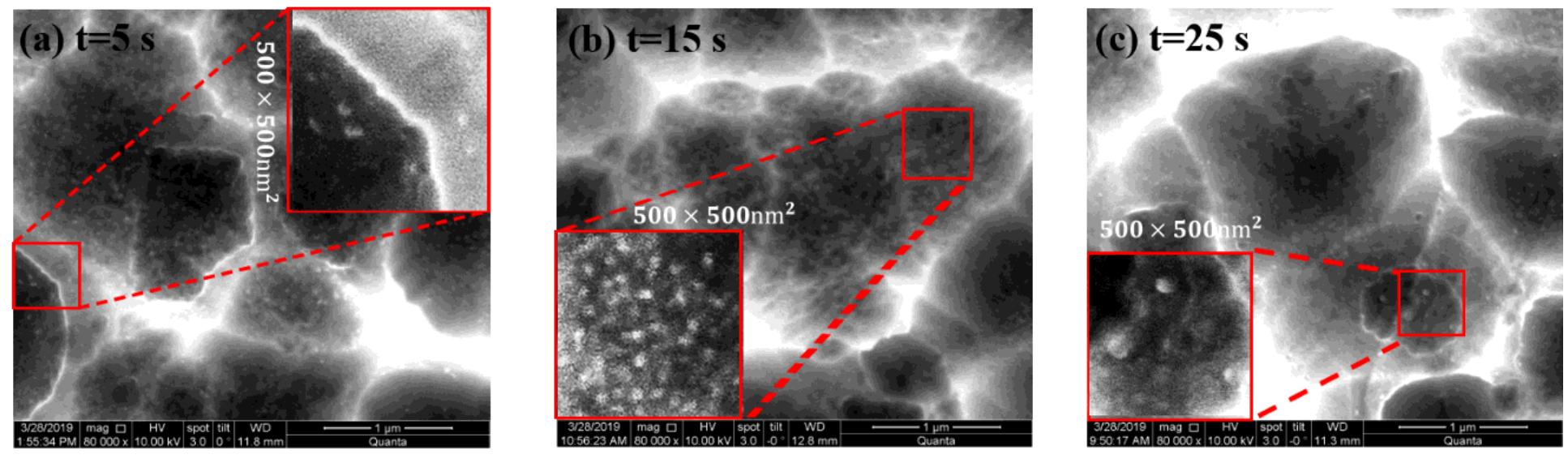

\section{Figure 3}

Stain-etched BSi with TiN nanoparticles at different deposition times (a) $5 \mathrm{sec}$; (b) $15 \mathrm{sec}$; (c) $25 \mathrm{sec}$

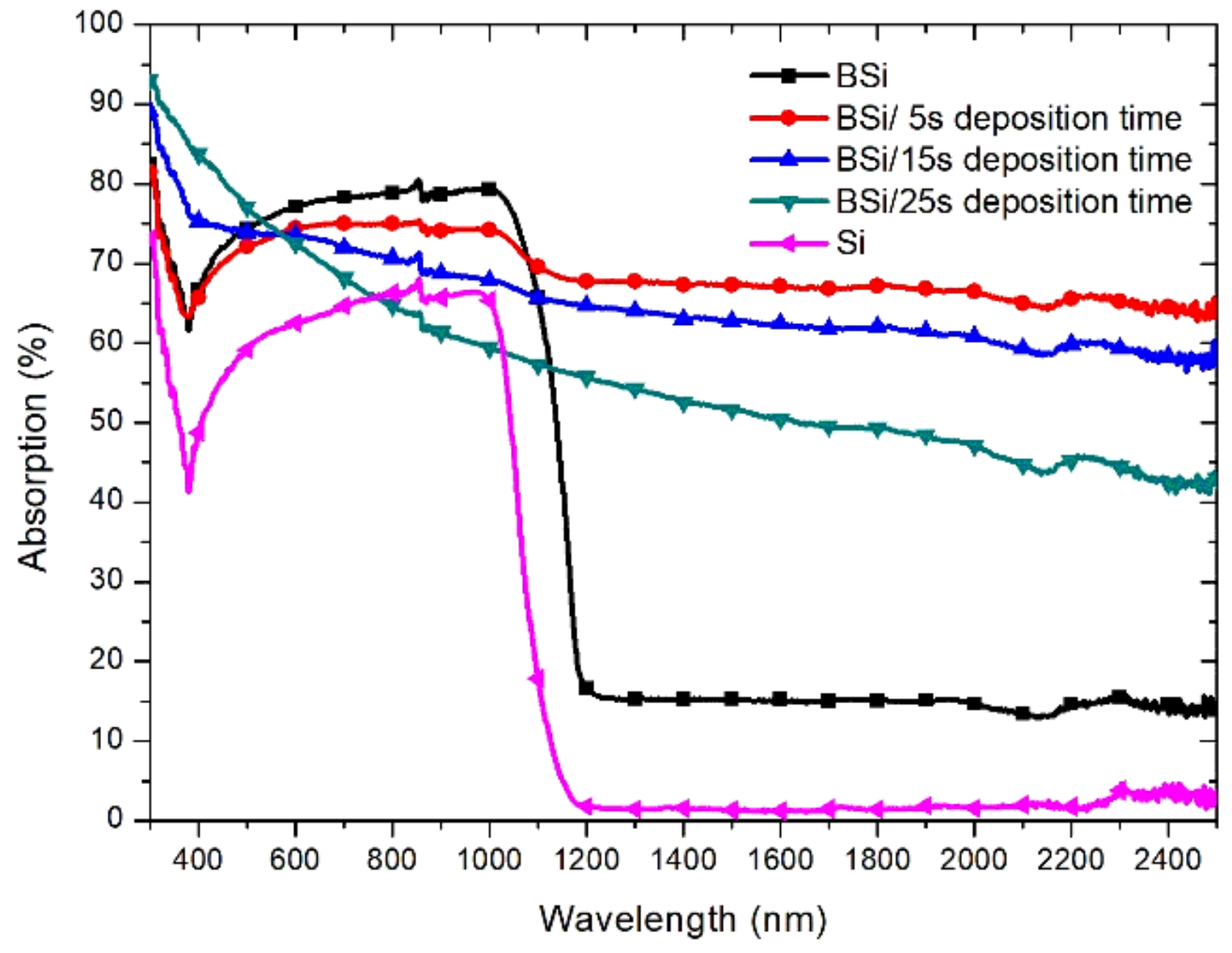

Figure 4 
Absorption curve of the BSi samples with different Ti targets deposition time, stain-etched BSi and planar-silicon

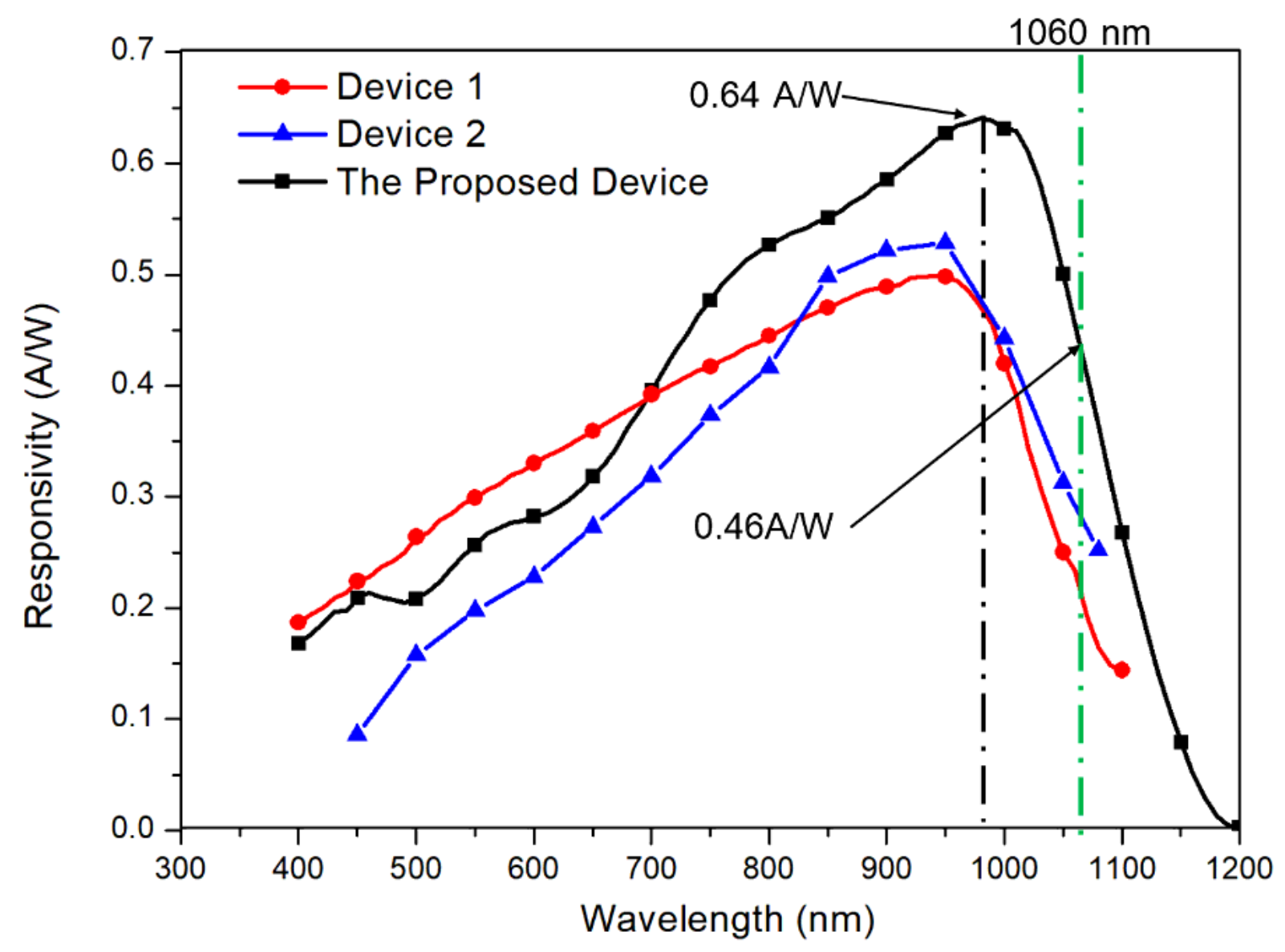

Figure 5

Responsivity spectra of three different detectors: device 1 from ref. [26], device 2 from ref. [27], and the proposed device based on the results of present paper 